INVESTIGACIÓN

\title{
SÍNDROME DEL BURNOUT Y CLIMA ORGANIZACIONAL EN EL INSTITUTO TÉCNICO DE FORMACIÓN PARA EL TRABAJO Y EL DESARROLLO HUMANO SURCOLOMBIANO
}

\author{
BURNOUT SYNDROME AND ORGANIZATIONAL CLIMATEIN \\ THE TECHNICAL TRAINING INSTITUTE FOR WORK \\ AND HUMAN DEVELOPMENT SURCOLOMBIAN
}

Juan Felipe Guzmán Pacheco*

Recibido: 14 de marzo de 2018

Evaluado: 23 de mayo de 2018

Aprobado: 11 junio de 2018

\section{RESUMEN}

El presente estudio de corte cuantitativo, diseño no experimental, de tipo descriptivo correlacional pretende identificar el nivel de prevalencia del síndrome de burnout en veinte docentes del municipio de Pitalito, Huila, y luego correlacionarla con las variables del clima organizacional. Los datos fueron analizados mediante el software estadístico SPSS (versión 21) y se obtuvieron los siguientes resultados: niveles bajos para las dimensiones de Cansancio emocional y Despersonalización, y para la Realización personal un nivel medio. La habilidad de afrontamiento más eficaz es el Afrontamiento activo. Por último, la dimensión organizacional más dinámica es la de Autonomía. Finalmente, se hallaron correlaciones positivas entre las variables anteriores.

Palabras clave: síndrome de burnout, habilidades de afrontamiento, clima organizacional, correlación, docentes. 


\section{INTRODUCCIÓN}

El síndrome de burnout consiste en "estar quemado, desgastado, exhausto y perder la ilusión por el trabajo" (Durán, 2013, p. 14). Es un padecimiento crónico provocado por el estrés laboral, que tiene efectos a largo plazo, es acumulativo y conlleva consecuencias negativas a nivel personal y organizacional.

A su vez, Geert Hofstede (1972, citado por Álvarez, 2006) indica que el clima organizacional está constituido por un ambiente humano y físico que se desarrolla día a día en contextos laborales. Este ambiente influye directamente en la satisfacción de los empleados y, por lo tanto, en la productividad (p. 21). También afecta el modo de compartamiento de los individuos en el ámbito laboral, lo que no depende solo de sus características personales, sino también de la forma en que éstos perciben su ambiente de trabajo, y los cambios y las transformaciones que acontecen en la empresa.

Los docentes refieren, en experiencias individuales $\mathrm{y}$ en entrevistas no estructuradas, en términos generales, que su actividad laboral se desarrolla con ambigüedad debido a las múltiples actividades que deben realizar con los padres de familia (o acudientes), los estudiantes y otros docentes. Además de sus actividades laborales, los docentes tienen la responsabilidad de su hogar y otras obligaciones con su comunidad. Todo esto añadido a que su vinculación dentro del Instituto es en calidad de empleo adicional, no es su actividad económica principal. Todo esto parece indicar que esta es una población vulnerable a algún tipo de estrés, considerando lo propuesto por Byrne (1999, ciado por Vinaccia y Alvarán, 2004).

Finalmente, este estudio pretende conocer la eficacia de las habilidades de afrontamiento y cómo las dimensiones organizacionales, que se presentan en esta población participante, inciden directa o indirectamente en el nivel del síndrome de burnout y si hay algún nivel de estrés en los docentes del Instituto Técnico Surcolombiano.

\section{DESARROLLO}

Dentro de la investigación se planteó la siguiente pregunta orientadora: ¿cuáles son los niveles del síndrome de burnout y cuál es su correlación con habilidades de afrontamiento y clima organizacional en los docentes del Instituto Técnico Surcolombiano? Adicional a esto, se plantearon los siguientes objetivos de investigación: identificar los niveles de burnout en los docentes del instituto, analizar las habilidades de afrontamiento de los docentes en el Instituto Técnico Surcolombiano, determinar las dimensiones de clima organizacional y establecer la correlación entre las habilidades de afrontamiento, el clima organizacional y el síndrome de burnout. 
Según Moreno et al. (2005) el síndrome de burnout se entiende como "una respuesta al estrés laboral crónico que conlleva la vivencia de encontrarse emocionalmente agotado, el cual comienza cuando el trabajador se siente emocionalmente incapaz de seguir encontrando satisfacción en su trabajo". Por esta razón, la reacción del empleado tiende a ser el distanciamiento de las fuentes que le generan dicho malestar, el desarrollo de actitudes y sentimientos negativos hacia las personas con las que se trabaja y, a su vez, la experiencia de ideas y sentimientos de baja realización tanto profesional como personal.

Por su parte, se considera que el síndrome de quemarse en el trabajo (burnout) es "un trastorno adaptativo y crónico que se asocia con el inadecuado afrontamiento de las demandas psicológicas, alterando la calidad de vida de la persona que lo presenta" (Gutiérrez et al., 2006). En este sentido, el síndrome de burnout reviste gran importancia, pues no sólo afecta la calidad de vida de los empleados, sino también el clima organizacional.

En el 2000, la Organización Mundial de la Salud (OMS) lo ha consideró un riesgo laboral, que afecta con más frecuencia a las profesiones que exigen mantener contacto directo con las personas, tales como: enfermeros, médicos, trabajadores sociales, profesores, psicólogos, policías, odontólogos, entre otros.
Entre los signos de alarma del síndrome de burnout se encuentra: la negación en la que la persona afectada es la última en aceptar que tiene el problema; el aislamiento en el que el empleado termina alejándose de sus familiares y amigos; la ansiedad o el temor de que algo malo le va a suceder; la depresión, que es uno de los síntomas más peligrosos ya que puede llevar al suicidio; la culpabilidad que es la idea de que lo que le pasa a la persona afectada es un castigo; las cargas excesivas de trabajo en personas que aceptan varios turnos en el trabajo. Estos son los principales signos de alarma, entre los que también se puede mencionar: sentimientos constantes de ira, cambios de personalidad, consumo de sustancias psicoactivas, entre otros (Gutiérrez et al. 2006).

Al respecto, el Maslach Burnout Inventory (MBI) es el instrumento que se ha utilizado con más frecuencia para evaluar el síndrome de burnout. Este fue creado por Cristina Maslach en 1986 y presenta una manera de cuantificar y de diagnosticar este síndrome. El cuestionario tiene varias versiones aplicables a diferentes poblaciones con diferentes idiomas y se ha mostrado que es un instrumento confiable y válido. Por estas razones es el que, en la actualidad, se usa con mayor frecuencia para medir la intensidad de este síndrome (Moreno et al. 2005). El cuestionario mide tres dimensiones: Cansancio o agotamiento emocional, que se 
caracteriza por la disminución y la pérdida de los recursos emocionales como resultado de excesivas demandas psicológicas y emocionales. También se desarrollan actitudes y sentimientos negativos hacia quienes atiende en su profesión o área de trabajo.

\section{METODOLOGÍA}

El presente estudio tiene un diseño metodológico cuantitativo, de tipo descriptivo correlacional, no experimental. La población estuvo conformada por 40 docentes vinculados al instituto. Sin embargo, la muestra fueron 20 docentes del Instituto Técnico Surcolombiano. La selección de dicha muestra se hizo con un método no probabilístico conveniencia, debido a la cercanía y al contacto con la dirección y la coordinación de talento humano del establecimiento educativo. Los participantes firmaron un consentimiento informado para el desarrollo de este estudio y se encuentran en edades comprendidas entre los 18 y los 60 años.

\section{INSTRUMENTOS.}

Se usaron dos instrumentos: el Maslach Burnout Inventory (MBI) presentado por Maslach y Jackson, es un instrumento en el que se le plantea al sujeto una serie de enunciados sobre los sentimientos y pensamientos en relación con su interacción con el trabajo. Es el instrumento que se ha utilizado en la mayoría de los estudios e investigaciones sobre el síndrome de burnout, que en Colombia fue validado por la Universidad de Antioquia en 2004.

El segundo instrumento fue el Inventario de clima organizacional de Koys y Decottis (1991). Este está constituido por 40 ítems. Sin embargo, para la presente investigación se emplearon 23 ítems. Los participantes respondieron a cada elemento en un formato de respuesta tipo Likert de cinco puntos, a saber: Muy de acuerdo $=5$, De acuerdo $=4$, No estoy seguro $=3$, En desacuerdo $=2$, Totalmente en desacuerdo $=1$. Seis elementos expresados en palabras de forma negativa son valorados de forma inversa. Las valoraciones de las escalas se obtienen mediante la suma total de los valores de los cinco elementos de cada escala. (Chiang Vega, Salazar Botello y Núñez).

\section{RESULTADOS}

Para la dimensión de Agotamiento emocional se hizo un hallazgo del 13,33. Esto indica un nivel bajo en esta dimensión, pues es inferior al 18 según el estandarizado. Para la dimensión de Despersonalización se halla un nivel de 4.41, lo que indica un bajo nivel al ser un puntaje menor que el estandarizado (5). Finalmente, para la dimensión de Autorrealización se halla una puntuación de 37.4 lo que permite ver que existe un nivel intermedio de autorrealización. 
La dimensión de Agotamiento emocional del burnout reveló una correlación positiva con la dimensión de Despersonalización con un nivel de significancia del 99\%. Esto indica que, a mayor agotamiento emocional mayor despersonalización, sugerido por la misma encuesta. De la misma manera, se encontró una relación significativa con la dimensión de Presión en dimensiones de clima organizacional con un nivel de significancia del 95\%. Esto indica que hay una fuerte incidencia entre correlaciones significativas.

Con respecto a la dimensión de Autorealización del burnout, se identificó una correlación positiva con la dimensión de Autonomía en un nivel de significancia del 99\%. Esto indica que a mayor Autorrealización mayor Autonomía. También se correlacionó positivamente con la dimensión de Equidad con un nivel de significancia del $99 \%$. Esto indica que, a mayor Logro mayor Equidad. Finalmente, se correlacionó positivamente y de manera significativa con la dimensión de cohesión con un nivel de significancia del 95\%.

La dimensión de Despersonalización de burnout se correlacionó positivamente con la dimensión de Presión de clima organizacional con un nivel de significancia del 99\%. Esto indica que hay una fuerte incidencia entre las variables. También se identificó una correlación significativa con la dimensión de Apoyo, dimensión de clima organizacional, con un nivel de significancia del $95 \%$, que indica que hay incidencia entre variables. Finalmente, se obtuvo una correlación significativa y positiva con la dimensión de reconocimiento, en un nivel de significancia del $95 \%$ y obteniendo así la inferencia entre variables.

Se obtuvo también una correlación significativa en la dimensión de Confianza con un nivel de significancia del 99\%. Esto indica una incidencia significativa entre variables. Otra dimensión con la que se encontró un nivel de correlación importante fue con la de Presión con una significancia del $99 \%$. Esto indica que las variables son directamente proporcionales entre sí. La dimensión de clima organizacional obtuvo una correlación significativa con la dimensión de Confianza, con un nivel de significancia del $99 \%$ lo que permite entender la dimensión de incidencia entre variables.

También se encontró una correlación positiva y significativa con la dimensión de Presión con un nivel de significancia del $99 \%$ que comprueba la incidencia entre variables. La dimensión de Cohesión se correlacionó significativamente con la dimensión de Reconocimiento con un nivel de significancia del $99 \%$ lo que indica que, a mayor cohesión mayor reconocimiento. 
La dimensión de clima organizacional Confianza se correlaciona positivamente con la de Apoyo de clima organizacional, con un nivel de significancia del $99 \%$ lo que indica que su relación es directamente proporcional. De igual manera se correlaciona positivamente con la dimensión de Reconocimiento con un nivel de significancia del $99 \%$ lo que indica el alto nivel de incidencia entre las variables. De igual manera, se correlacionó positivamente con la dimensión de Equidad con un nivel de significancia del $99 \%$ y en donde se comprueba su alto nivel de incidencia entre variables.

La dimensión del clima organizacional de Presión se correlacionó positivamente con la dimensión de apoyo con un nivel de significancia del 99\% lo que justifica la incidencia entre variables de manera creciente y decreciente. De igual manera de reveló una correlación significativa con la dimensión de Reconocimiento en un nivel del 99\% justificando la incidencia proporcional para ambas variables. También se evidenció una relación significativa con la dimensión de Innovación con un nivel de significancia del $99 \%$ lo que indica que a mayor presión mayor Innovación, algo inusual por su correlación. También se evidenció un nivel de correlación positiva con la dimensión de Equidad con un nivel de significancia del 99\% lo que indica una incidencia entre variables.
El clima organizacional en Apoyo evidenció una correlación significativa con la dimensión Apoyo, con un nivel de significancia del 99\% lo que indica que a mayor Apoyo mayor Autonomía, significante correlación positiva. De igual manera se reveló una correlación significativa con la dimensión de Cohesión con un nivel de significancia del 99\% lo que indica que las variables tienes una fuerte incidencia entre sí. También se evidenció una correlación significativa y positiva con la dimensión de innovación a un nivel de significancia del 99\% justificando así, la incidencia entre sí y para ambas variables. También, la dimensión de Apoyo se correlacionó positivamente con la variable de equidad con un nivel de significancia del $99 \%$ en donde se evidencia la relación directamente proporcional entre ambas variables.

La dimensión de Reconocimiento de clima organizacional presentó una correlación positiva con la dimensión de Apoyo con un nivel de significancia del 99\%. Esto indica que a mayor reconocimiento mayor apoyo a los docentes. También se correlacionó positivamente con la dimensión de Equidad con un nivel de significancia del $99 \%$ lo cual justifica un alto grado de incidencia entre las variables correlacionadas. También se correlacionó positiva y significativamente con la dimensión Autonomía con un nivel de significancia de $99 \%$ lo que indica 
que las variables están unidas de una manera proporcional. También se presentó una correlación positiva ante la dimensión de Innovación con nivel de significancia del $99 \%$ en donde se presenta, en las dos variables, el mismo grado de variabilidad.

La dimensión de clima organizacional de Equidad presentó una correlación significativa con la dimensión de Autonomía con un nivel de significancia del $99 \%$ lo que permite evidenciar un alto nivel de incidencia entre las variables. De igual manera, la dimensión de Equidad se relacionó positivamente con la dimensión de Cohesión con un nivel de significancia del $99 \%$ en donde se permite evidenciar el alto nivel de incidencia entre las variables.

La dimensión de Innovación de clima organizacional presentó una relación significativa con la dimensión de Autonomía con un nivel de significancia del $99 \%$ que indica un alto nivel de incidencia entre variables. De la misma manera se correlaciona significativamente con la dimensión de Confianza con un nivel de significancia del $99 \%$ justificando así, el alto nivel de incidencia que hay entre ambas variables. También se relacionó positivamente con la dimensión de cohesión con un nivel de significancia del $99 \%$ lo que indica que, a mayor innovación mayor cohesión. Finalmente, se correlacionó positivamente con la dimensión de Equidad con un nivel de incidencia del $99 \%$ que indica una fuerte incidencia entre ambas variables.

\section{DISCUSIÓN}

Los datos de burnout, que se presentaron en la sección anterior, se analizaron a partir de la compilación y esquematización de cada dimensión hecha por Rodríguez et al (2011): "Agotamiento emocional: Bajo < 19; Medio 19 - 26 y Alto $\geq 27$, Despersonalización: Bajo: < 6; medio: 6-9 y alto $10 \geq$. Realización personal: bajo $\leq 31$ nivel medio $31-38$ y alto $\geq 39$ " $A$ partir de lo anterior, se puede inferir que en los docentes del municipio de Pitalito, Huila, los niveles son bajos ante cualquier dimensión del burnout. Siguiendo a Gil y Peiro (2000), el estrés manejado en el ámbito laboral de los docentes no tiene incidencia en su vida personal, ya que sus habilidades de afrontamiento y las dimensiones organizacionales, resultan eficaces para no permitir el impacto del estrés a partir del agotamiento emocional y la despersonalización, por el contrario, fortaleciendo la autorrealización de los docentes en su contexto laboral.

Las características de sentirse emocionalmente cansado, incapaz, aislarse de sus compañeros y la dejación intermitente del trabajo es muy baja, que es lo propuesto por Moreno et al. (2005). De igual manera, estos 
resultados refutan la teoría de Gutiérrez Celis (2006) de la incidencia negativa en la salud mental de la población en cuestión. Ante los factores de riesgo del burnout propuestos por Gutiérrez et al. (2006), entre los que se pueden mencionar: ser jóven, de género femenino, soltero, con sobrecarga de trabajo, con rasgos de personalidad como idealista, optimista, con deseos de prestigio y de mayores ingresos económicos, entre los cuales el más importante es el de sobrecarga laboral, ha incidido en la anidación del síndrome en ninguna de las variables de la población analizada.

En cuanto al objetivo de la investigación, se pudo identificar el nivel de burnout en los docentes. Este nivel, según los resultados, es bajo. Esto es un indicador de que las habilidades de afrontamiento y las dimensiones de clima organizacional inciden directamente en la anulación del burnout. De esta manera en la presente investigación se comprueba que a menor nivel de burnout mayor habilidades de afrontamiento y un clima organizacional adecuado. "Las características del ambiente que facilitan que el trabajador desarrolle sentimientos de éxito se corresponden con las características de las organizaciones que ayudan a prevenir el síndrome de burnout: un alto nivel de desafío, autonomía, control, retroalimentación de los resultados y apoyo social del supervisor y de los compañeros"
(Cherniss, 1993). La anterior teoría fortalece los resultados de la presente investigación en cuanto al nivel emocional y actitudinal de la población abordada. Con respecto a las habilidades de afrontamiento más eficaces en los docentes, se puede inferir que, por la modalidad de trabajo, se fortalece la dimensión de afrontamiento activo y la religión son las habilidades son las más empleadas. En cuanto a clima organizacional, las dimensiones más eficaces es la Autonomía difuminando especialmente las dimensiones del burnout.

Se esperaba la correlación obtenida entre las variables. Esto indica que la relación entre las variables es directamente proporcional y que las variables dependientes inciden positivamente en la variable independiente. En cuanto a las habilidades de afrontamiento, se encontró que son estrategias de respuesta ante algún evento y clima organizacional, entendido como un contexto de autorealización personal, haciendo lo que se quiere y valorando positivamente los resultados laborales.

\section{CONCLUSIONES}

Los niveles de burnout en los docentes del Instituto Técnico Surcolombiano, a partir de la implementación del $\mathrm{MBI}$, arrojó el siguiente consolidado general por dimensiones: Agotamiento Emocional, 13,33 (bajo), Autorrealización 37,24 (intermedio), 
Despersonalización 4,41 (bajo). Se encontraron algunas excepciones a partir de la caracterización demográfica: según el estado civil (divorciado) se evidenció un bajo nivel de autorrealización $(41,71 \mathrm{n}=7)$; según el número de Hijos (ninguno) autorrealización baja $(41,88$ $n=8)$ y, finalmente, en cuanto a la dimensión de Despersonalización, hay un nivel intermedio en la característica demográfica de estado civil $(9 n=2)$, al igual que la característica demográfica de nivel educativo (profesional) $(7,89 n=9)$.

Para clima organizacional y $\mathrm{MBI}$ : Despersonalización 99\%, Presión 95\%, Autonomía 99\%, Equidad 99\%, Cohesión 95\%, Presión 95\%, Apoyo 95\%, Reconocimiento $95 \%$. Esto indica que las variables generales de clima organizacional, en el el ámbito laboral, afectan directamente las dimensiones del burnout.

\section{REFERENCIAS}

Gutiérrez, G., Celis, M., Moreno, S., Farias, F. \& Suárez, J. (2006). Síndrome de Burnout. Arch. Neurocien., 11 (4), 305-309.

Moreno, B., Rodríguez, A., Garrosa, E. \& Morante, M. (2005). Breve historia del Burnout a través de sus instrumentos de evaluación. En Quemarse en el trabajo: 11 perspectivas del burnout (161-183). Madrid, España: Editorial Egido.

Noriega, V. \& Pria, M. (2011). Instrumento para evaluar el clima organizacional e $\mathrm{n}$ los grupos de control de vectores. Revista cubana salud pública, 37 (2), 116-122.

Sandoval duran, d. n. (4 de 05 de 2013). google. recuperado el 24 de 05 de 2017, de google: http:// stadium.unad.edu.co/preview/unad.php?url=/ bitstream/10596/2176/1/protecto.pdf 